\section{BIOLOGY AND MATHEMATICS}

\author{
Jordi Bascompte \\ Estación Biológica de Doñana, CSIC
}

RESUMEN: La biología se ha convertido en la nueva "física" de las matemáticas, una de las áreas con mayores aplicaciones. Las matemáticas, por su parte, han proporcionado herramientas y metáforas muy poderosas para abordar la increible complejidad de los sistemas biológicos. Esto ha permitido la génesis de marcos conceptuales sólidos. En este artículo resumo algunas de las aplicaciones más exitosas de las matemáticas a la biología que van desde la genética de poblaciones a la biología del desarrollo y las redes de interacciones ecológicas.

PALABRAS CLAVE: Formación de patrón, dinámica no-lineal, redes complejas, dinámica de poblaciones, genética de poblaciones, estructura de comunidades, desarrollo, epidemiología, biología teórica.

Charles Darwin, the father of the modern theory of evolution through natural selection, once complained that "I have deeply regretted that I did not proceed far enough at least to understand something of the great leading principles of mathematics; for men thus endowed seem to have an extra sense." While it is well appreciated the huge contribution of mathematics to physics, its role in biology is not so generally acknowledged. However, mathematics has been of paramount importance in the understanding of life sciences, and this importance will even increase in the near future. This essay provides an opinionated and biased appreciation of such a contribution from mathematics to biology and vice versa.

If physics was the battlefield where mathematics was meeting the real world during the $19^{\text {th }}$ century, biology became the area of greatest intellectual challenges and applications for mathematics in the $20^{\text {th }}$ century. As nicely written by Joel E. Cohen, mathematics became to biology what the microscope represented a few centuries ago: a tool to approach and interpret a new and fascinating world. Certainly, it was not until the invention of the microscope in the late $17^{\text {th }}$ century when a world invisible to the naked eye was suddenly discovered. Similarly, mathematics is a way to describe complex systems. Think for example in the complexity of the human brain, with its huge number of neurons and
ARBOR Ciencia, Pensamiento y Cultura CLXXXIII 725 mayo-junio (2007) 347-351 ISSN: 0210-1963

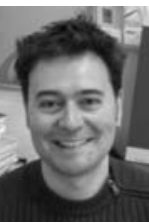

ABSTRACT: Biology has become the new "physics" of mathematics, one of the areas of greatest mathematical applications. In turn, mathematics has provided powerful tools and metaphors to approach the astonishing complexity of biological systems. This has allowed the development of sound theoretical frameworks. Here, I summarize some of the most significant contributions of mathematics to biology, ranging from population genetics, to developmental biology, and to networks of species interactions.

KEY WORDS: Pattern formation, non-linear dynamics, complex networks, population dynamics, population genetics, community structure, development, epidemiology, theoretical biology.

interactions, or in the thousands of species in a tropical forest interacting in complex ways. Biology represents challenges into the complex, and math may help us to approach such a complexity. Mathematics represents a way to extract straightforward consequences of a series of assumptions: if $x$ is assumed, then $y$ is derived. This does not only allow us to think clearly, but to perform mathematical experiments in systems in which real experiments are impossible.

Arguably, the Hardy-Weinberg law in population genetics is equivalent with Newton's First Law: if no force is applied into a population (e.g., natural selection, migration), the population will remain in a genetic equilibrium, that is, gene frequencies will not change in a population from one generation to another. Population genetics is one of the fields with a highest mathematical development. Partly this is due to the extraordinary long time scale in which evolutionary forces take place. It is impossible to observe changes in gene frequencies through time. Oftentimes, what we have is a temporal slice, and from these snapshots we are to infer a process. A rich mathematical development by the fathers of the modern evolutionary synthesis allowed us to merge Darwin's theory on natural selection with quantitative genetics. The resulting theory has been a fascinating intellectual contribution to understanding 
what forces shape live in this planet and why there is so much genetic variation in nature. Mathematics has thus been the glue bringing together evolution and genetics.

Ecology has, to some extent, followed the track of population genetics with some delay. Even when ecology has been mainly a descriptive science, the pioneering work by Lotka, Volterra, Nicholson, Bailey and others first introduced mathematical representations of temporal changes in populations. Their models were based on either partial differential equations or equations in difference. While the former describe time as a continuous variable, the latter use discrete representations, e.g., number of generations. In any case, models of population ecology describe how a population changes though time as a function of its own density and/or the density of its predator, parasite, or a competing species. Even when these are just cartoons of reality, these population models were extremely important in understanding complex real phenomena such as population cycles. By means of these models one could first explore mechanisms contributing to the stability of populations and communities.

Today, ecologist face the pressing needs arising from the accelerating human influence in the Biosphere. These needs require a predictive theory, the kind of theory that can guide us, for example, in understanding the consequences of habitat loss and fragmentation on biodiversity. Here is one example of the type of understanding mathematics has provided to ecology. Habitat loss is the leading cause of biodiversity decline. However, we do not have a theoretical framework to predict its consequences on populations and communities. Our intuition would tell us that we would still find a population, albeit of a shrinking size, as far as some fraction of the original habitat is left. These mathematical models, however, predicted that beyond a threshold of habitat destruction, the population goes extinct. At these critical values, populations and communities are at the edge of extinction: there is no relationship anymore between the intensity of a perturbation and the magnitude of its consequences. Two and two do no longer add four as Robert May once noted. Predicting these points of no return is very important in conservation.

A particular type of extremely simplified ecosystem is that composed by the interaction between viruses and the immune system. Epidemiology has been another important field in biology where mathematics has allowed a rich conceptual development. Let's consider, for example, the spread of smallpox within a human population. Epidemiological models describe the temporal dynamics in the number of hosts infected and make predictions such as the existence of a critical population size beyond which the diseases fades away. Similarly, epidemiological models predict a critical fraction of hosts to be vaccinated for the eradication of the diseases. These thresholds are essentially identical to the extinction thresholds described above in the context of habitat loss. Thus, mathematics can guide the design of efficient vaccination programs.

A notorious example of virus is the HIV, the infectious agent causing AIDS. These RNA-based viruses mutate at an incredible rate to escape the immune system. This is the reason of their high success. The immune system evolves to recognize the exact nature of an invader and to counterbalance it, but if the virus keeps changing, it escapes this defensive system. Mathematical models of distributions of virus strains called quasispecies have shown the existence of a mutation rate threshold. Beyond such a threshold, called catastrophe of error, an increase of the mutation rate induces the loss of the genetic information. It is like the phase transition separating liquid and solid states in physics. It represents the transition from sequences with biological information to random sequences. These fast mutating viruses are thus at the edge. The same biological process that has made them so elusive to classical epidemiological treatments such as vaccines can provide keys for their eradication. Just push a little bit forward its mutation rates and the virus will collapse. John Holland and colleagues have demonstrated experimentally this by using several mutants to increase the mutation rate of the vesicular stomatitis virus. As a result, the virus becomes non-infectious.

Developmental biology is another field in biology that has benefited from mathematics. How can the process leading from one cell to a complex embryo be explained from basic principles? This was a total mystery reflecting a complete divorce between the $19^{\text {th }}$ century physics focusing on systems at thermodynamic equilibrium, and so showing temporal evolutions towards disorder, and the dynamics towards increasing order and complexity characteristic of life. The great mathematician Alan Turing provided the first theoretical approximation to solve this apparent paradox. 
Turing is famous for at least two other contributions, namely inventing the Turing machine, i.e., the precursor of modern computers, and breaking the Enigma code used by the German Nazis to encrypt communications during the Second World War. After these two previous contributions, Turing had the brilliant idea in 1952 of writing down a mathematical model describing the dynamics of two chemical species called morphogens. The rules are as follows. First, one morphogen called activator produces itself at a rate proportional to its abundance. This is a type of multiplicative, non-linear process very common in biology: the more activator, the faster is produced. Second, the activator also produces a second morphogen called inhibitor, which in turn inhibits the former. Third, both activator and inhibitor diffuse through space, although the inhibitor does it at a faster rate. In summary, the system is described by local production and long-range inhibition. Turing showed that starting from a uniform spatial distribution of both morphogens, some random fluctuations will be amplified. At the end, there will be patches with a high concentration of activator surrounded by empty areas. We have gone from a homogeneous distribution to a heterogeneous one. Imagine that this heterogeneous distribution of activator determines the formation of a head on one extreme, where the concentration of activator is beyond the average, and a tail on the other side, where the activator's concentration is below the average. Symmetry has been broken though a bifurcation of the homogeneous solution. Structure has appeared. The size of the spatial domain in which these morphogens diffuse determines how many such bifurcations can be accommodated.

Elegant extensions of the Turing model have been proposed to explain multiple examples of pattern formation in development such as the pigmentation in the coat of some mammals. The great developmental biologist Pere Alberch, in collaboration with George Oster, James Murray, and others, used this type of mathematical formulation in combination with beautiful experiments in which the size of an amphibian extremity could be manipulated trough mutagens. As this size was progressively decreased, skeletal elements were deleted in a sequential order, mirroring natural variation in related species. Alberch and colleagues were able to show that evolution takes place through minor changes of a conserved developmental program. Self-organization plays a very important role that cannot be anticipated by focusing exclusively on genes. These self- organizing spatial patterns show discrete bifurcations following a well-defined sequence, and so natural selection has only a limited set of possibilities to choose from.

It seems through this brief description that the flow of ideas has always gone from mathematics to biology. Although this is the case in the majority of examples, there are also some cases in which the influence is the other way around. That is, biology has also made a contribution to mathematics. An illustrative case is that of deterministic chaos. This mathematical theory challenged a solid assumption arising from the Newtonian paradigm. Certainly, Newtonian mechanics had represented a triumph of science. Newton's laws were able to describe the dynamics of celestial bodies and, more spectacularly, to make powerful predictions. Given an individual condition, let's say the position and speed of a comet right now, and the deterministic law of gravity, one can predict the position of such a comet 100 or 1,000 years from now. Or in the past, because for that matter the system is reversible, one can move the tape recorder either backwards or forwards. Newton started a new way to deal with nature in which deviations from what should be expected could be used to make specific claims. An example was the prediction of the planet Neptune on the basis of the modification of the gravitational field of the other planets. Newton's contribution was so important, that Alexander Pope proposed the following epitaph for Newton, who died in 1727: "Nature and nature's laws lay hid in night: God said, let Newton be! And all was light." One can argue who has been the second most important scientist in history, but the first position undoubtedly belongs to Isaac Newton.

In the previous scheme, however, there is a small caveat: we cannot know perfectly an initial condition. The atmospheric temperature at a place and a time, for example, is a number with infinite decimal points. What we do is to round this number. Let's say we take five decimal points. This extremely small mistake remains small in systems like the ones studied by Newton. Thus, if the arrival of the comet after 1,000 years is predicted with an error of five decimal points, nobody would claim the theory is not good. In this type of systems, small errors remain small through time. This is because these are linear systems, that is, systems in which variables add one to another. However, lots of interesting systems such as the weather and biological systems are non-linear. In this case, variables

ARBOR CLXXXIII 725 mayo-junio [2007] 347-351 ISSN: 0210-1963

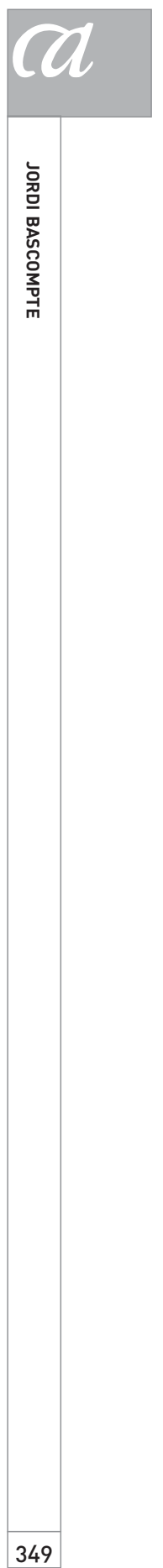


do not add but multiply each other or are raised to an exponent. The consequence of this is that errors now will not remain small. They will grow exponentially through time. The paradigmatic example is weather prediction. It is not that scientists do not understand the dynamics of fluids. They do so as well as they understand Newton's laws. The problem is that weather is described by nonlinear equations. A very small mistake after, let's say 10 days, will become so large that prediction just does not work for long temporal windows. It is no longer true that knowledge implies prediction. The temporal dynamics of systems like weather show random-like behavior similar to what we would expect for a stochastic system, but they are generated by totally deterministic systems described by a small number of variables. This behavior was named deterministic chaos.

There were several contributions to the mathematics of deterministic chaos. Some were certainly done by mathematicians, but others by climatologists such as Edward Lorenz, and by the theoretical ecologists Robert May. Lorenz was working on a simplified model of weather and found that after running a simulation a second time he obtained a different output. Since his system of three differential equations was absolutely deterministic, he thought he had made a mistake. Finally he realized there was a tiny difference in the two initial conditions. He had encountered deterministic chaos and its strong dependence on initial conditions.

Robert May, trained as a physicist, shifted to population biology and become one of the leading theoreticians in ecology and epidemiology. May co-discovered chaos by studying a simple, deterministic model of an ecological population. It was a difference equation or logistic map. The simplest model one can conceive in population ecology. It deterministically specifies the population density at the next generation given the population density at the current generation. May iterated the model starting from a given population density. The behavior of this model was found to be very dependent on the value of the growth rate, a measure of per-capita fertility. For low growth rates, the population evolves towards a constant value, what mathematicians would call a steady state. Once the population reaches this value, nothing else happens. No big surprise so far: a simple model shows a simple behavior. If now we slightly increase the growth rate, the model evolves towards period-two cycles. Now the population will repeat itself every other generation. Although something more interesting than before, this is still a simple behavior. If this parameter is further increased, cycles with higher periods $(4,8,16, \ldots)$ arise until a specific growth rate is reached. Now the population fluctuates without an apparent pattern. It never repeats itself. This is deterministic chaos.

May's work inspired mathematicians who developed a theory about the existence of some universal properties of deterministic chaos. The type of period-doubling route to chaos May had found for his logistic map, was proved to be absolutely general. Similar non-linear models and experimental systems were found to have the same properties. In this case, an important mathematical contribution was originated from the domain of ecology.

The mathematics of deterministic chaos embraces one type of biological complexity: dynamic complexity. Another source of complexity in biological systems has to do with their large number of interacting elements. We already mentioned the examples of the human brain and the food webs, graphical depictions of who eats whom in an ecological community. These highly complex systems have eluded analytical tractability. A branch of mathematics analyzes graphs described by nodes linked by edges. This framework is currently quite popular, and finds important applications both in biology and in other fields. In biology, the study of networks of genetic interactions opens the path towards a better understanding of gene regulation and the treatment of some genetic disorders. In ecology, considering the network of species interactions provides a community-wide approximation to the spreading of human-induced perturbations such as overfishing. New generation computers, with an increasing power can eventually simulate the dynamics of these extremely rich systems. Computer software based on mathematical algorithms has also been used to represent complex data such as the human genome. A full genome is like a text with millions of letters, and one cannot just plot this huge amount of gross information. To have a chance to visualize this and seek patterns, one needs intelligent software.

Mathematics, in sum, provides useful approximations to the real world. This has the advantage of controlling all confounding factors, isolating the interesting variables, 
and allowing to perform experiments we would not be able to do in nature. And this exercise can ultimately shed some light into the complexities of biological systems. Simple models are the "perfect crystals" of biology, a kind of benchmark or reference where one can get straight-

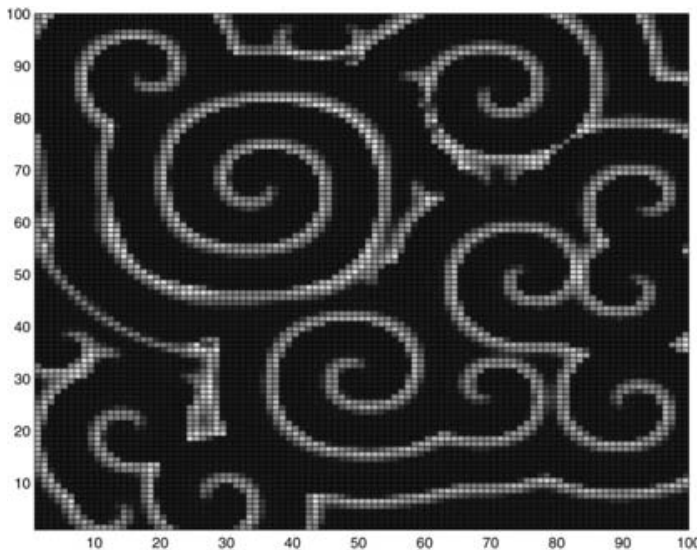

Figure 1. Simple lattice models of interacting populations create self-organizing spatial patterns such as these traveling waves. These spatial models are based on local non-linear dynamics and dispersal to nearby patches. The figure represents a snapshot for a specific time step; the shadow of each spatial cell represents the density of one of the populations forward insight. As we walk through the challenging complexities of brain dynamics or the consequences of global change on ecological communities, mathematics will become an even more powerful language to speak about life.

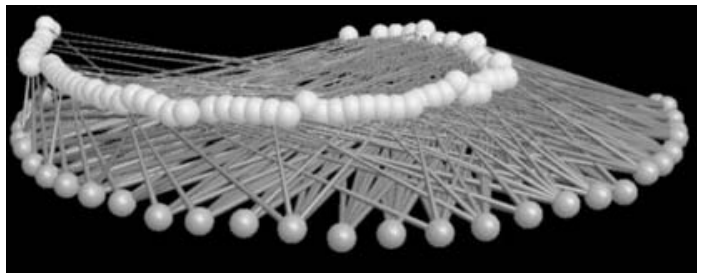

Figure 2. The architecture of biodiversity: Species within an ecosystem form complex networks of dependency which architecture largely determines the robustness of these communities to perturbations. The figure represents a pollination community in the Arctic. Bottom and top nodes represent plant and insect species, respectively: A link between a plant-animal pair indicates that the latter pollinates the former
Recibido: 13 de diciembre de 2006 Aceptado: 15 de diciembre de 2006

\section{REFERENCES}

Cohen, J. E. (2004): "Mathematics is biology's next microscope, only better; biology is mathematics' next physics, only better", PloS Biol, 2 (12): e439.

Goodwin, B. C. (1994): How the Leopard Changed Its Spots; The Evolution of Complexity, Weidenfeld and Nicolson, London.

Gillespie, J. H. (1998): Population Genetics: A Concise Guide, The John Hopkins University Press, Baltimore.
May, R. M. (2004): "Uses and abuses of mathematics in biology", Science 303: 790-793.

Murray, J. D. (1989): Mathematical Biology. Springer-Verlag, Berlin.

Solé, R. V. and Bascompte, J. (2006): Self-Organization in Complex Ecosystems; Princeton University Press.

Stewart, I. (1989). Does God Play Dice? The Mathematics of Chaos. Basil BlackweII, Oxford. 